\title{
Symmetry-preserving contact interaction model for heavy-light mesons
}

\author{
F. E. Serna, M. A. Brito and G. Krein \\ Instituto de Física Teórica, Universidade Estadual Paulista \\ Rua Dr. Bento Teobaldo Ferraz, 271 - Bloco II, 01140-070 São Paulo, SP, Brazil
}

\begin{abstract}
We use a symmetry-preserving regularization method of ultraviolet divergences in a vector-vector contact interaction model for low-energy QCD. The contact interaction is a representation of nonperturbative kernels used Dyson-Schwinger and Bethe-Salpeter equations. The regularization method is based on a subtraction scheme that avoids standard steps in the evaluation of divergent integrals that invariably lead to symmetry violation. Aiming at the study of heavy-light mesons, we have implemented the method to the pseudoscalar $\pi$ and $K$ mesons. We have solved the Dyson-Schwinger equation for the $u, d$ and $s$ quark propagators, and obtained the bound-state Bethe-Salpeter amplitudes in a way that the Ward-Green-Takahashi identities reflecting global symmetries of the model are satisfied for arbitrary routing of the momenta running in loop integrals.
\end{abstract}

Keywords: Regularization of ultraviolet divergences, Ward-Green-Takahashi identities, Dyson-Schwinger and Bethe-Salpeter equations PACS: 11.10.St, 11.15.Tk, 11.30.-j, 14.40.Df, 14.40.Lb, 24.85.+p

\section{INTRODUCTION}

Contact fermionic interactions find widespread applications in hadron physics with Nambu-Jona-Lasinio (NJL) [1] type of models. A great deal of qualitative insight on the phenomenon of hadron mass generation via dynamical chiral symmetry breaking $(\mathrm{D} \chi \mathrm{SB})$ and the role of the pions as the associated (quasi) Goldstone bosons has been gleaned from such models - for reviews, see Refs. [2, 3, 4, 5]. The lack of confinement and nonrenormalizabilty are the major weaknesses of contact fermionic models. The nonrenormalizability carries with it the danger of introducing gross violations of global symmetries due to the regularization procedure; ambiguities arising from momentum shifts in divergent integrals are the main cause of the problems. Despite well known, practitioners at large inexplicably ignore these problems. However, a new perspective has emerged recently with the implementation $[6,7,8]$ of a confining, symmetry-preserving treatment of a vector-vector contact interaction as a representation of the gluon's two-point Schwinger function used in kernels of Dyson-Schwinger (DS) equations. By introducing a mechanism that ensures the absence of quark production thresholds, a feature of a confining theory, and embedding the interaction in a global-symmetry-preserving, rainbow-ladder truncation framework of the DS and BS equations $[9,10]$, the scheme has enhanced the capacity of a contact interaction to describe in a unified manner a diverse array of nonperturbative QCD phenomena - for a recent review, see Ref. [11]

In this communication we examine the contact-interaction model within the perspective of a regularization scheme that allows to separate symmetry-offending parts in BS amplitudes in a way independent of choices of momentum routing in divergent integrals, so that the Ward-Green-Takahashi (WGT) identities reflecting global symmetries of the model are preserved by the regularization. The scheme is inspired in a method introduced in Refs. [12, 13] to manipulate divergent Feynman integrals without specification of an explicit regulator. The method has been used with the NJL model in vacuum $[14,15]$ and at finite temperature and baryon density $[16,17,18]$. We formulate the problem generically with the aim of using it for heavy-light mesons, and assess its reliability presenting numerical results for the $\pi$ and $K$ mesons.

\section{DS AND BS EQUATIONS AND WGT IDENTITIES}

We start with a brief review of the basic elements of the contact-interaction scheme introduced in Ref. [6]. We consider the inhomogeneous BSE for a quark and antiquark state of total momentum $P$ (here and in the following we omit 
renormalization constants)

$$
\left[\Gamma_{\mathscr{M}}(k ; P)\right]_{A B}=\mathscr{M}_{A B}+\int_{q}[K(k, q ; P)]_{A C, D B}\left[S\left(q_{+}\right) \Gamma_{\mathscr{M}}(q ; P) S\left(q_{-}\right)\right]_{C D},
$$

where $\int_{q} \equiv \int d^{4} q /(2 \pi)^{4}, \mathscr{M}$ gives the Dirac spinor structure of the state, $K(q, k ; P)$ is the fully amputated quarkantiquark scattering kernel; $A, B, \cdots$ denote collectively color, flavor, and spinor indices; $q_{ \pm}=q \pm \eta_{ \pm} P$, with $\eta_{+}+\eta_{-}=$ 1 and $q$ is the relative momentum. $S(k)$ is the dressed-quark propagator given by a DSE; for a given flavor $f$ the general form of this DSE is

$$
S_{f}(k)^{-1}=i \gamma \cdot k+m_{f}+\int_{q} g^{2} D_{\mu v}(k-q) \frac{\lambda^{a}}{2} \gamma_{\mu} S_{f}(q) \frac{\lambda^{a}}{2} \Gamma_{v}^{f}(q, k),
$$

where $m_{f}$ is the current-quark mass. Here we will be interested in the flavor-nonsinglet axial-vector $\Gamma_{5 \mu}^{l h}(k ; P)$ and pseudoscalar $\Gamma_{\mathrm{PS}}^{l h}(k ; P)$ amplitudes for a quark of flavor $l$ and antiquark of flavor $h$, for which $\mathscr{M}_{5 \mu}=\gamma_{5} \gamma_{\mu}$ and $\mathscr{M}_{P S}=\gamma_{5}$ respectively. Translation invariance requires that physical observables cannot depend on the choice of the momentum routing in quark propagators in Eq. (1); that is, physical results must be independent of $\eta_{ \pm}$.

Associated with $\Gamma_{5 \mu}^{l h}$ is the Ward-Green-Takahashi (WGT) identity

$$
P_{\mu} \Gamma_{5 \mu}^{l h}(k ; P)=S_{l}^{-1}\left(k_{+}\right) i \gamma_{5}+i \gamma_{5} S_{h}^{-1}\left(k_{-}\right)-i\left(m_{l}+m_{h}\right) \Gamma_{\mathrm{PS}}^{l h}(k ; P),
$$

where $\Gamma_{P S}^{l h}(k ; P)$ is the pseudoscalar vertex. Pseudoscalar meson bound states are obtained from the solution of the homogeneous equation for $\Gamma_{\mathrm{PS}}^{l h}(k ; P)$

$$
\left[\Gamma_{\mathrm{PS}}^{l h}(k ; P)\right]_{A B}=\int_{q}[K(k, q ; P)]_{A C, D B}\left[S_{l}\left(q_{+}\right) \Gamma_{\mathrm{PS}}^{l h}(q ; P) S_{h}\left(q_{-}\right)\right]_{C D},
$$

where here $A, B, \cdots$ denote color and spinor indices only. The general form of the of $\Gamma_{\mathrm{PS}}^{l h}(k ; P)$ is

$$
\Gamma_{\mathrm{PS}}^{l h}(k, P)=\gamma_{5}\left[i E_{\mathrm{PS}}^{l h}+\gamma \cdot P F_{\mathrm{PS}}^{l h}+\gamma \cdot k G_{\mathrm{PS}}^{l h}+\sigma_{\mu v} k_{\mu} P_{v} H_{\mathrm{PS}}^{l h}\right],
$$

where $E_{\mathrm{PS}}^{l h}, F_{\mathrm{PS}}^{l h}, \cdots$ are functions of $k, P$ and $k \cdot P$. The meson mass, $m_{\mathrm{PS}}$, is the eigenvalue $P^{2}=-m_{\mathrm{PS}}^{2}$ that solves Eq. (4).

The contact-interaction scheme introduced in Ref. [6] amounts to make the following replacements in Eq. (2) and the construction of scattering kernels of all BSEs

$$
g^{2} D_{\mu v}(k-q) \rightarrow \frac{4 \pi \alpha_{I R}}{m_{G}^{2}} \delta_{\mu v} \quad \text { and } \quad \Gamma_{\mu}^{a} \rightarrow \frac{\lambda^{a}}{2} \gamma_{\mu},
$$

where $m_{G}$ is a gluon mass-scale and $\alpha_{I R}$ is a fitting parameter. This means that the scattering kernel in all BSEs is

$$
[K(k, q ; P)]_{A C, D B}=-\frac{4 \pi \alpha_{I R}}{m_{G}^{2}}\left(\frac{\lambda^{a}}{2} \gamma_{\mu}\right)_{A C}\left(\frac{\lambda^{a}}{2} \gamma_{\mu}\right)_{D B} .
$$

A feature of the momentum independence of the contact interaction is that the DS and BS equations become nonrenormalizable. This means that mass-scale parameters introduced with the regularization of divergent integrals cannot be removed from the calculations and need to be fixed phenomenologically. Another feature, as mentioned previously, is that regularization of divergent integrals carries the danger of symmetry violation, in that WGT identities, like the one in Eq. (3), are not maintained, even when Poincaré-invariant regularization schemes are employed.

Let us consider the DSE and the homogeneous pseudoscalar BSE, Eqs. (2) and (4), with the contact interaction. In this case, $S_{f}(k)^{-1}=i \gamma \cdot k+M_{f}$, with

$$
M_{f}=m_{f}+\frac{64 \pi \alpha_{I R}}{3 m_{G}^{2}} \int_{q} \frac{M_{f}}{q^{2}+M_{f}^{2}} .
$$

In addition, $G_{\mathrm{PS}}^{l h}=H_{\mathrm{PS}}^{l h}=0$, so the Bethe-Salpeter amplitude Eq. (5) takes the form

$$
\Gamma_{\mathrm{PS}}^{l h}(P)=\gamma_{5}\left[i E_{\mathrm{PS}}^{l h}(P)+\frac{1}{2 M_{l h}} \gamma \cdot P F_{\mathrm{PS}}^{l h}(P)\right]
$$


where $M_{l h}=M_{l} M_{h} /\left(M_{l}+M_{h}\right)$. Then, the BSE can be written in the matrix form

$$
\left[\begin{array}{c}
E_{\mathrm{PS}}^{l h}(P) \\
F_{\mathrm{PS}}^{l h}(P)
\end{array}\right]=\frac{4 \pi \alpha_{I R}}{3 m_{G}^{2}}\left[\begin{array}{cc}
\mathscr{K}_{l h}^{E E} & \mathscr{K}_{l h}^{E F} \\
\mathscr{K}_{l h}^{F E} & \mathscr{K}_{l h}^{F F}
\end{array}\right]\left[\begin{array}{c}
E_{\mathrm{PS}}^{l h}(P) \\
F_{\mathrm{PS}}^{l h}(P)
\end{array}\right],
$$

where

$$
\begin{aligned}
\mathscr{K}_{l h}^{E E} & =-\int_{q} \operatorname{Tr}\left[\gamma_{5} \gamma_{\mu} S_{l}\left(q_{+}\right) \gamma_{5} S_{h}\left(q_{-}\right) \gamma_{\mu}\right], \\
\mathscr{K}_{l h}^{E F} & =\frac{i}{2 M_{l h}} \int_{q} \operatorname{Tr}\left[\gamma_{5} \gamma_{\mu} S_{l}\left(q_{+}\right) \gamma_{5} \gamma \cdot P S_{h}\left(q_{-}\right) \gamma_{\mu}\right], \\
\mathscr{K}_{l h}^{F E} & =\frac{2 M_{l h}^{2}}{P^{2}} \mathscr{K}_{l h}^{E F} \\
\mathscr{K}_{l h}^{F F} & =\frac{1}{P^{2}} \int_{q} \operatorname{Tr}\left[\gamma_{5} \gamma \cdot P \gamma_{\mu} S_{l}\left(q_{+}\right) \gamma_{5} \gamma \cdot P S_{h}\left(q_{-}\right) \gamma_{\mu}\right] .
\end{aligned}
$$

Here, the trace is over Dirac indices. All integrals in Eqs. (8) and (11)-(14) are ultraviolet divergent; the divergences are quadratic and logarithm. The vast majority of applications within NJL models ignore the pseudo vector component $F_{\mathrm{PS}}^{l h}(P)$; in doing so, this leads to the random-phase-approximation (RPA) for the BS equation.

\section{SYMMETRY-PRESERVING SUBTRACTION SCHEME}

The issue of symmetry violation can be exposed examining the momenta running in the quark propagators; they are $q_{+}=q+k_{1}$ and $q_{-}=q+k_{2}$, with $k_{1}=\eta_{+} P$ and $k_{2}=-\eta_{-} P$. The momenta $k_{1}$ and $k_{2}$ are arbitrary, as while satisfying $\eta_{+}+\eta_{-}=1$, the $\eta_{ \pm}$are otherwise arbitrary. However, to maintain translation invariance, the integrals must depend only on the relative momentum $k_{1}-k_{2}$ or, equivalently, the integrals must not depend on $\eta_{ \pm}$, they can depend only in the combination $\eta_{+}+\eta_{-}=1$. The dependence on $k_{1}-k_{2}$ only is also crucial for maintaining the WGT identities, like the one in Eq. (3).

The traditional way of handling within NJL-type of models integrals like those in Eqs. (11)-(14) is as follows: after evaluating the traces, a choice for $\eta_{+}$and $\eta_{-}$is made and Feynman parameters are used to combine in a single term the product $\left(q_{+}^{2}+M_{l}^{2}\right)\left(q_{-}^{2}+M_{h}^{2}\right)$ in the denominator; then, after a momentum shift to eliminate the angle $q \cdot P$ in the denominator, the integral over $q$ is performed. In making the momentum shift, changes in the integration limits are ignored. Invariably, results depend upon the choices made for $\eta_{ \pm}$; in particular, the value of the leptonic decay constants, which play a key role in the normalisation of the pion BSE, depend upon the choices made for $\eta_{ \pm}$. Some regularization-independent results, like the Goldberg-Treiman relation at the quark level and the Gell-Mann-OakesRenner relation, can be obtained after using the gap equation to eliminate the quadratic divergences.

The subtraction scheme of Refs. $[12,13,14,15,16,17,18]$ is based on the repeated use of the identity

$$
\begin{aligned}
\frac{1}{q_{ \pm}^{2}+M^{2}} & =\left(\frac{1}{q_{ \pm}^{2}+M^{2}}-\frac{1}{q^{2}+M^{2}}\right)+\frac{1}{q^{2}+M^{2}} \\
& =\frac{1}{q^{2}+M^{2}}-\frac{q_{ \pm}^{2}-q^{2}}{\left(q^{2}+M^{2}\right)} \frac{1}{\left(q_{ \pm}^{2}+M^{2}\right)}
\end{aligned}
$$

Assuming a Poincaré invariant regularization for the integrals in Eqs. (11)-(14), subtractions are performed for each of the propagators $S_{l}\left(q_{+}\right)$and $S\left(q_{-}\right)$, the number of which is dictated by the requirement that a finite integral is obtained - note that while the original denominator goes as $1 / q^{2}$ for $q \rightarrow \infty$, the last term in Eq. (15) goes as $1 / q^{3}$ for $q \rightarrow \infty$. We illustrate the procedure in detail for the $\mathscr{K}_{l h}^{E E}(P)$ kernel. Evaluation of the trace in Eq. (11) leads to

$$
\mathscr{K}_{l h}^{E E}=16 \int_{q}^{\Lambda} \frac{q_{+} \cdot q_{-}+M_{l} M_{h}}{\left(q_{+}^{2}+M_{l}^{2}\right)\left(q_{-}^{2}+M_{h}^{2}\right)},
$$

where $\Lambda$ indicates the mass scale associated with the regularization. Next, subtracting thrice each of the denominators, $\mathscr{K}_{l h}^{E E}$ can be written as a sum of three kinds of terms: quadratic and logarithmically divergent integrals that are 
independent of $\eta_{ \pm}$, symmetry violating terms that are proportional to $\eta_{+}$and $\eta_{-}$, and a finite integral also independent of $\eta_{ \pm}$. Explicitly, they are given by the following expressions:

$$
\begin{aligned}
\mathscr{K}_{\mathrm{PS}}^{E E}= & 8\left\{\left[\eta_{+}^{2} A_{\mu v}\left(M_{l}^{2}\right)+\eta_{-}^{2} A_{\mu v}\left(M_{h}^{2}\right)\right] P_{\mu} P_{v}+I_{\text {quad }}\left(M_{l}^{2}\right)+I_{\text {quad }}\left(M_{h}^{2}\right)\right. \\
& \left.-\left[P^{2}+\left(M_{h}-M_{l}\right)^{2}\right]\left[I_{\log }\left(M_{l}^{2}\right)-Z_{0}\left(M_{l}^{2}, M_{h}^{2}, P^{2} ; M_{l}^{2}\right)\right]\right\},
\end{aligned}
$$

where $A_{\mu v}\left(M^{2}\right), I_{\text {quad }}\left(M^{2}\right)$ and $I_{\log }\left(M^{2}\right)$ are the divergent integrals (when $\Lambda \rightarrow \infty$ )

$$
\begin{gathered}
A_{\mu \nu}\left(M^{2}\right)=\int_{q}^{\Lambda} \frac{4 q_{\mu} q_{v}-\left(q^{2}+M^{2}\right) \delta_{\mu v}}{\left(q^{2}+M^{2}\right)^{3}} \\
I_{\text {quad }}\left(M^{2}\right)=\int_{q}^{\Lambda} \frac{1}{q^{2}+M^{2}}, \quad I_{\log }\left(M^{2}\right)=\int_{q}^{\Lambda} \frac{1}{\left(q^{2}+M^{2}\right)^{2}},
\end{gathered}
$$

and $Z_{0}\left(M_{l}^{2}, M_{h}^{2}, P^{2} ; M_{l}^{2}\right)$ is a finite integral given by

$$
Z_{0}\left(M_{l}^{2}, M_{h}^{2}, P^{2} ; M_{l}^{2}\right)=\frac{1}{(4 \pi)^{2}} \int_{0}^{1} d z \ln \left[\frac{P^{2} z(1-z)-\left(M_{l}^{2}-M_{h}^{2}\right) z+M_{l}^{2}}{M_{l}^{2}}\right] .
$$

It is important to note that to arrive at these results, no momentum shifts were made in divergent integrals. The other amplitudes, $\mathscr{K}_{l h}^{E F}, \mathscr{K}_{l h}^{F E}$, and $\mathscr{K}_{l h}^{F F}$ are given by similar integrals [19].

Note that Eq. (17) makes it clear that whatever choice made for $\eta_{ \pm}$, unavoidably translation symmetry is broken, unless the regularization scheme leads to $A_{\mu v}\left(M^{2}\right)=0$. Let us next examine how choices of $\eta_{ \pm}$lead to violation of the WGT identity in Eq. (3). One needs to deal with integrals of the form

$$
\begin{aligned}
\int_{q}^{\Lambda} \frac{q_{\mu}}{q_{ \pm}^{2}+M^{2}}= & \mp \eta_{ \pm} P_{\mu} I_{\text {quad }}\left(M^{2}\right) \pm \frac{1}{3} \eta_{ \pm}^{3}\left[P^{2} A_{\mu \rho}\left(M^{2}\right) P_{\rho}-P_{\mu} A_{\rho \sigma}\left(M^{2}\right) P_{\rho} P_{\sigma}\right] \\
& \mp \eta_{ \pm} B_{\mu \rho}\left(M^{2}\right) P_{\rho} \mp \frac{1}{3} \eta_{ \pm}^{3} C_{\mu \rho \sigma \lambda}\left(M^{2}\right) P_{\sigma} P_{\rho} P_{\lambda},
\end{aligned}
$$

where $B_{\mu \nu}\left(M^{2}\right)$ and $C_{\mu v \rho \sigma}\left(M^{2}\right)$ are the new structures

$$
B_{\mu v}\left(M^{2}\right)=\int_{q}^{\Lambda} \frac{2 q_{\mu} q_{v}-\left(q^{2}+M^{2}\right) \delta_{\mu v}}{\left(q^{2}+M^{2}\right)^{2}}, \quad C_{\mu v \rho \sigma}\left(M^{2}\right)=\int_{q}^{\Lambda} \frac{c_{\mu v \rho \sigma}\left(q, M^{2}\right)}{\left(q^{2}+M^{2}\right)^{4}},
$$

with

$$
c_{\mu v \rho \sigma}\left(q^{2}, M^{2}\right)=24 q_{\mu} q_{v} q_{\rho} q_{\sigma}-4\left(q^{2}+M^{2}\right)\left(\delta_{\mu v} q_{\rho} q_{\sigma}+\text { perm. } v \sigma \rho\right) .
$$

Using these integrals in the WGT identity, Eq. (3), one obtains

$$
0=\left(M_{l}-m_{l}\right)+\left(M_{h}-m_{h}\right)-\frac{64 \pi \alpha_{I R}}{3 m_{G}^{2}}\left\{I_{\text {quad }}\left(M_{l}^{2}\right)+I_{\text {quad }}\left(M_{h}^{2}\right)+\left[\eta_{+}^{2} A_{\mu v}\left(M_{l}^{2}\right)+\eta_{-}^{2} A_{\mu v}\left(M_{h}^{2}\right)\right]\right\} P_{\mu} P_{v}
$$

and

$$
0=\int_{q}^{\Lambda}\left(\frac{q_{+} \cdot P}{q_{+}^{2}+M^{2}}-\frac{q_{-} \cdot P}{q_{-}^{2}+M^{2}}\right) \sim \text { terms proportional to } \eta_{ \pm}\left(A_{\mu \nu}, B_{\mu \nu}, C_{\mu \nu \rho \sigma}\right) .
$$

We see that for arbitrary momentum routing in the loop integrals, i.e. for arbitrary values of $\eta_{ \pm}$satisfying $\eta_{+}+\eta_{-}=1$, the subtraction scheme allows to identify in a systematic way symmetry offending terms; they are the integrals $A_{\mu v}, B_{\mu \nu}$ and $C_{\mu v \rho \sigma}$ in the equations above. A consistent regularization scheme should make the integrals vanish automatically. Otherwise, the vanishing of the integrals must be imposed; in doing so, the regularization scheme 
becomes part of the model. Dimensional regularization and Pauli-Villars regularization are examples of schemes that lead to $A_{\mu v}=0, B_{\mu v}=0$, and $C_{\mu \nu \rho \sigma}=0$. Removing the symmetry offending terms, the kernels $\mathscr{K}_{l h}^{E E}, \mathscr{K}_{l h}^{E F}, \mathscr{K}_{l h}^{F E}$, and $\mathscr{K}_{l h}^{F F}$ become free from ambiguities and symmetry-preserving.

Let us make contact with the scheme of Ref. [6]. For $M_{l}=M_{h}=M$ and $\eta_{+}=1$ and $\eta_{-}=0$, which is the choice made in Ref. [6], Eq. (25) becomes

$$
\int_{q}^{\Lambda}\left(\frac{q_{+} \cdot P}{q_{+}^{2}+M^{2}}-\frac{q \cdot P}{q^{2}+M^{2}}\right)=0
$$

This is precisely Eq. (15) of Ref. [6]. Using Eq. (21) for this integral, one obtains

$$
0=\int_{q}^{\Lambda}\left(\frac{q_{+} \cdot P}{q_{+}^{2}+M^{2}}-\frac{q \cdot P}{q^{2}+M^{2}}\right)=P_{\mu}\left[B_{\mu \nu}\left(M^{2}\right)+\frac{1}{3} C_{\mu \nu \rho \sigma}\left(M^{2}\right) P_{\rho} P_{\sigma}+\frac{1}{3} P_{\mu} A_{\rho \nu}\left(M^{2}\right) P_{\rho}-\frac{4}{3} P^{2} A_{\mu \nu}\left(M^{2}\right)\right] P_{v}
$$

that is

$$
B_{\mu v}\left(M^{2}\right)+\frac{1}{3} C_{\mu \nu \rho \sigma}\left(M^{2}\right) P_{\rho} P_{\sigma}+\frac{1}{3} P_{\mu} A_{\rho v}\left(M^{2}\right) P_{\rho}-\frac{4}{3} P^{2} A_{\mu v}\left(M^{2}\right)=0 .
$$

In the quiral limit, $P=0$, this leads to

$$
B_{\mu v}\left(M^{2}\right)=0=\delta_{\mu \nu} \int_{\Lambda} \frac{d^{4} q}{(2 \pi)^{4}} \frac{\frac{1}{2} q^{2}+M^{2}}{\left(q^{2}+M^{2}\right)^{2}}
$$

which is Eq. (17) of Ref. [6]. This result makes it clear that our scheme, besides being in agreement with Ref. [6] for the particular choice of $\eta_{ \pm}$, it is also more general, as it is valid for arbitary values $\eta_{ \pm}$.

\section{NUMERICAL RESULTS}

In order assess the reliability of the subtraction scheme, we apply it to the calculation of the masses and electroweak decay constants of the $\pi$ and $K$ mesons. The mass of the meson is obtained from Eq. (10); it is an eigenvalue problem which has a solution at $P^{2}=-m_{\mathrm{PS}}^{2}$. We use the canonical normalization condition for the BS amplitudes, namely

$$
1=\left.\frac{\partial}{\partial P^{2}} \Pi_{\mathrm{PS}}^{l h}(Q, P)\right|_{Q=P}
$$

with

$$
\Pi_{\mathrm{PS}}^{l h}(Q, P)=6 \int \frac{d^{4} q}{(2 \pi)^{4}} \operatorname{Tr}\left[\bar{\Gamma}_{\mathrm{PS}}^{h l}(-Q) S_{l}\left(q+k_{1}\right) \Gamma_{\mathrm{PS}}^{l h}(Q) S\left(q+k_{2}\right)\right]
$$

where $\bar{\Gamma}_{\mathrm{PS}}^{h l}(-Q)=\left[C^{-1} \Gamma_{\mathrm{PS}}^{l h}(-Q) C\right]^{T}=\Gamma_{\mathrm{PS}}^{l h}(-Q)$. The electroweak decay constant of the meson, $f_{\mathrm{PS}}$, can be expressed in terms of normalized amplitudes $E$ and $F$ in the form

$$
f_{\mathrm{PS}}=\left.\frac{6}{M_{l h}}\left[E_{\mathrm{PS}}(P) \mathscr{K}_{\mathrm{PS}}^{F E}+F_{\mathrm{PS}}(P) \mathscr{K}_{\mathrm{PS}}^{F F}\right]\right|_{P^{2}=-m_{\mathrm{PS}}^{2}} .
$$

We compare results with Ref. [20], where the scheme of Ref. [6] was used for the $K$ meson. We use proper-time regularization for $I_{q u a d}^{\Lambda}\left(M_{f}\right)$. In the BS amplitudes where $I_{q u a d}^{\Lambda}\left(M_{f}\right)$ appears, we use the gap equation (8), and for $I_{l o g}^{\Lambda}\left(M_{f}\right)$ use the identity

$$
I_{\log }^{\Lambda}\left(M_{f}\right)=-\frac{d I_{q u a d}^{\Lambda}\left(M_{f}\right)}{d M_{f}^{2}}
$$

On the other hand, for the subtraction mass scale we have used the light mass $M_{l}^{2}$ and finite integrals like $Z_{0}\left(M_{h}^{2}, M_{l}^{2}, P^{2} ; M_{h}^{2}\right)$ are performed without imposing a regulator. 
We use the same parameters employed in Ref. [20]: $\alpha_{I R}=0.93 \pi, m_{G}=0.8 \mathrm{GeV}, m_{u}=m_{d}=0.007 \mathrm{GeV}$ and $m_{s}=0.160 \mathrm{GeV}, \tau_{I R}^{2}=(0.24 \mathrm{GeV})^{2}$ and $\tau_{U V}^{2}=(0.905 \mathrm{GeV})^{2}$. The last two parameters are the infrared and ultraviolet cutoff parameters of the proper-time regularization. The solution of the gap equation leads for the constituent quark masses $M_{u}=M_{d}=0.367 \mathrm{GeV}$, and $M_{s}=0.525 \mathrm{GeV}$. Table I presents our results and those of Ref. [20] (indicated with a star).

TABLE 1. Masses and electroweak decay constants $\pi$ and $K$ mesons. The four first columns show the results obtained using the subtraction scheme and the four last indicated with a star, are the results of Ref. [20].

\begin{tabular}{ccccccccc}
\hline Meson & $E$ & $F$ & $m_{\mathrm{PS}}$ & $f_{\mathrm{PS}}$ & $E^{*}$ & $F^{*}$ & $m_{\mathrm{PS}}^{*}$ & $f_{\mathrm{PS}}^{*}$ \\
\hline$\pi$ & 3.759 & 0.498 & 0.139 & 0.106 & 3.596 & 0.474 & 0.139 & 0.101 \\
$K$ & 3.984 & 0.632 & 0.494 & 0.115 & 3.864 & 0.591 & 0.493 & 0.107 \\
\hline
\end{tabular}

We see from Table I that the results obtained with the subtraction scheme compares well with those of Ref. [20]: the masses are almost identical in both approaches, and the decay constants from the subtraction are a little larger, but by less than $5 \%$. We recall that the differences in the approaches are that in the subtraction scheme the results are independent of the choice of $\eta_{ \pm}$, while those of Ref. [20] are for $\eta_{+}=1$ and $\eta_{-}=0$. Another difference is that the finite integral $Z_{0}\left(M_{h}^{2}, M_{l}^{2}, P^{2} ; M_{h}^{2}\right)$ is integrated without imposing ultraviolet or infrared cutoffs. There is no difficulty in using an infrared cutoff in the finite integrals to avoid unphysical quark-antiquark thresholds in amplitudes.

\section{CONCLUSIONS AND PERSPECTIVES}

We examined the contact-interaction model introduced in Ref. [6] within the perspective of a regularization scheme that allows to separate symmetry-offending parts in Bethe-Salpeter amplitudes in a way independent of choices of momentum routing in divergent integrals. In doing so, the Ward-Green-Takahashi (WGT) identities reflecting global symmetries of the model are preserved by the regularization. Symmetry-offending parts of the amplitudes, the integrals $A_{\mu \nu}, B_{\mu \nu}$ and $C_{\mu \nu}$, can be neatly separated. In general, a cutoff regularization scheme leads to nonzero values for the symmetry-offending integrals, while Pauli-Villars or dimensional regularization lead to the vanishing of the symmetryoffending integrals. In a nonrenormalizable model, like the contact-interaction model discussed here, the vanishing of $A_{\mu \nu}, B_{\mu \nu}$ and $C_{\mu \nu}$ must be imposed in an ad hoc manner.

Our aim is to apply the subtraction scheme to heavy-light mesons, where the heavy quark is much heavier than the light quark, like in the $D$ and $B$ mesons. When the masses are not much different, one can show that in the RPA approximation to the BS equation, the heavy-light pseudoscalar meson mass $m_{\mathrm{PS}}^{2}$ and corresponding electroweak decay constant $f_{\mathrm{PS}}$ can be expressed in terms of the light-quark condensate $\langle\bar{q} q\rangle$ and the pion decay constant $f_{\pi}$ as

$$
\begin{aligned}
m_{\mathrm{PS}}^{2} & \simeq\left(M_{h}-M_{l}\right)^{2}-m_{h} \frac{M_{l}}{M_{h}} \frac{\langle\bar{q} q\rangle}{f_{\pi}^{2}} \\
f_{\mathrm{PS}} & \simeq \frac{1}{2}\left(1+\frac{M_{h}}{M_{l}}\right) f_{\pi}
\end{aligned}
$$

These expressions are valid for $M_{h} \leq \Lambda$, where $\Lambda$ is the regularization mass scale in the divergent integrals $I_{\text {quad }}$ and $I_{\log }$. Eq. (35) shows very clearly that $f_{\pi}<f_{K}<f_{D}$, which is the correct ordering of their experimental values. Using the traditional cutoff regularization scheme, in which no attention is given to symmetry-offending terms in amplitudes, one obtains [21] $f_{\pi} \simeq f_{K}$ and $f_{D}<f_{\pi}$. Of course, Eqs. (34) and (35) are not strictly applicable to this case, but they show the trend one can expect when using the subtraction scheme. Because of the large diferences between the masses of the quarks running in the loops, the application of the subtraction method in this case is nontrivial and needs adaptations - results will be presented elsewhere [19]. 


\section{ACKNOWLEDGMENTS}

The work of M.A.B. and F.E.S. were financed by doctoral scholarships by Coordenação de Aperfeiçoamento de Pessoal de Nível Superior - CAPES and Conselho Nacional de Desenvolvimento Científico e Tecnológico - CNPq, respecytively. The work of G.K. was supported in part by Conselho Nacional de Desenvolvimento Científico e Tecnológico - CNPq, Grant No. 305894/2009-9, and Fundação de Amparo à Pesquisa do Estado de São Paulo FAPESP, Grant No. 2013/01907-0.

\section{REFERENCES}

1. Y. Nambu and G. Jona-Lasinio, Phys. Rev. 122, 345 (1961).

2. U. Vogl and W. Weise, Prog. Part. Nucl. Phys. 27, 195 (1991).

3. S. Klevansky, Rev. Mod. Phys. 64, 649 (1992).

4. T. Hatsuda and T. Kunihiro, Phys. Rept. 247, 221 (1994).

5. J. Bijnens, Phys. Rept. 265, 369 (1996).

6. L. X. Gutiérrez-Guerrero, A. Bashir, I. C. Cloët and C. D. Roberts, Phys. Rev. C 81, 065202 (2010).

7. H. L. L. Roberts, C. D. Roberts, A. Bashir, L. X. Gutiérrez-Guerrero and P. C. Tandy, Phys. Rev. C 82, 065202 (2010).

8. H. L. L. Roberts, A. Bashir, L. X. Gutiérrez-Guerrero, C. D. Roberts and D. J. Wilson, Phys. Rev. C 83, 065206 (2011).

9. H. J. Munczek, Phys. Rev. D 52, 4736 (1995).

10. A. Bender, C. D. Roberts and L. von Smekal, Phys. Lett. B 380, 7 (1996).

11. I. C. Cloet and C. D. Roberts, Prog. Part. Nucl. Phys. 77, 1 (2014).

12. O. A Battistel, PhD Thesis 1999, Universidade Federal de Minas Gerais, Brazil (unpublished).

13. O. A. Battistel and M. C. Nemes, Phys. Rev. D 59, 055010 (1999).

14. O. A. Battistel, G. Dallabona and G. Krein, Phys. Rev. D 77, 065025 (2008) .

15. O. A. Battistel and G. Dallabona, Phys. Rev. D 80, 085028 (2009).

16. R. L. S. Farias, G. Dallabona, G. Krein and O. A. Battistel, Phys. Rev. C 77, 065201 (2008).

17. R. L. S. Farias, G. Dallabona, G. Krein and O. A. Battistel, Phys. Rev. C 73, 018201 (2006).

18. R. L. S. Farias, G. Krein, G. Dallabona and O. A. Battistel, Nucl. Phys. A 790, 332 (2007).

19. F. E. Serna , M. A. Brito and G. Krein, in preparation.

20. C. Chen, L. Chang, C. D. Roberts, S. M. Schmidt, S. Wan and D. J. Wilson, Phys. Rev. C 87, 045207 (2013).

21. D. Blaschke, P. Costa, and Yu. L. Kalinovsky, Phys. Rev. D 85, 034005 (2012). 